\title{
Characterization and Synthesis Mechanism of Nanosilver/PAMPS Composites by Microwave
}

\author{
Guocai Xu*, Shengtao Gao, Xiaoli Ji, Xiaomei Zhang \\ School of Chemical Engineering, Anhui University of Science and Technology, Huainan, China \\ Email: ${ }^{*}$ gcxu@aust.edu.cn
}

Received 3 December 2013; revised 3 January 2014; accepted 10 January 2014

Copyright (C 2014 by authors and Scientific Research Publishing Inc.

This work is licensed under the Creative Commons Attribution International License (CC BY). http://creativecommons.org/licenses/by/4.0/

(c) (i) Open Access

\begin{abstract}
Nanosilver/Poly(2-acrylamido-2-methylpropanesulfonate sodium (AMPS)) composites were synthesized with sliver nitrate solution containing AMPS monomer in situ by microwave radiation without addition of any reducer. The composites were characterized by means of UV-Vis, XRD, FTIR, TEM and XPS respectively. The results show that silver nanoparticles are dispersed highly and homogeneously in PAMPS matrix and possessed face-centered cubic structure. The morphology of nanosilver particles is not affected by microwave treatment and AMPS polymerization. XPS analysis reveals that there is an interaction among silver nanoparticles with nitrogen atoms and carbonyl oxygen atoms from AMPS polymer. Thermoanalysis proves that thermal stability of the PAMPS nanocomposites is decreasing with silver nanoparticles dispersed in the composites. The mechanism of silver ion reduced to nanosilver particles catalysized by PAMPS under microwave is discussed.
\end{abstract}

\section{Keywords}

Nano-Silver, PAMPS, Composite, Microwave

\section{Introduction}

Research activities on inorganic-polymer nanocomposite materials have an exponential growth due to many new characters, such as thermal, electrical, mechanical, optical properties, etc., which can be widely used in optics materials, electricity materials, biomedicine materials, information materials and other fields [1] [2].

Among the nanocomposites, especially metal-based materials, such as, silver-polymer nanocomposites, are of

*Corresponding author.

How to cite this paper: Xu, G.C., Gao, S.T., Ji, X.L. and Zhang, X.M. (2014) Characterization and Synthesis Mechanism of Nanosilver/PAMPS Composites by Microwave. Soft Nanoscience Letters, 4, 15-23.

http://dx.doi.org/10.4236/snl.2014.42003 
special interest [3] [4]. This is because nanoscale metal particles such as silver and gold provide a very exciting research field due to their interesting optical, electronic, magnetic, and catalytic properties [5]-[7]. Silver exhibits the highest electrical and thermal conductivities among all the metals and has applications in a variety of areas such as catalysis, conductive inks and pastes \& adhesives for electronic components, photonics and photography [8] [9].

As for syntheses of metal-based nanocomposites, several methods have been established for the preparation of silver-polymer nanocomposite, including physical techniques such as thermal evaporation, laser sputtering and layer-by-layer (LBL) assembly [10], and chemical techniques such as in-situ chemical reduction [11], sonochemical deposition [12], UV radiation reduction [13] and electroless plating [14], etc. But there are more or less defects in those synthetic technologies, such as using reductant, complex synthetic steps, difficult post-processing and so on. Microwave heating is a completely neoteric method to prepare nano-Ag/polymer composites.

Microwave radiation has the advantage of heating uniformly and making metal nanoparticles easily nucleate, and manifests the unique superiority in the preparation of nano-materials. Li [15], Angshuman [16], Yao [17] et al. synthesized nano-silver composites by microwave radiation, but they prepared nanosilver composites with polymer existing as protective agent, which restricted the selectivity of polymer as matrices of composite materials. PAMPS form in-situ polymerization has eximious dispersion, thermal stability and a lot of other special performance; it has a greatly protective effect on nanosize silver particles as matrix. Xu et al. [18] reported nano-Ag composite materials with AMPS copolymer as continues phases synthesized by ultrasonic. The synthesis of silver nanoparticles composites by simpler and easier synthetic routes continues to be a challenging area of research; the microwave reaction can be conducted at room temperature and at ambient pressure, and furthermore, the reaction is free of reducing agents. At the present time, there is no report on in-situ synthesis of nano-Ag/polymer composites by microwave radiation as far as our knowledge goes.

In this paper, nano-Ag/PAMPS composite was prepared by the silver nitrate solution containing AMPS monomer without reducer, in which silver ion was reduced to silver nanoparticles and the monomer was polymerized simultaneously by microwave radiation, and the polymers are considered to be better steric stabilizing agents for nano-metal to improve the stability and dispersion of nanosilver in polymer matrix. The nano-silver particles are highly and homogeneously dispersed in PAMPS polymer as continues phases of nanosilver/polymer composite; it simplifies the preparation process of nanocomposite materials and post-processing process of synthesis system because of inexistence of other matter. The interaction between silver nanoparticles and PAMPS polymer matrix is discussed. Specially, the mechanism of silver ion which is reduced to nano-silver particles catalyzed by PAMPS without metal-reducer under microwave is discussed.

\section{Experimental}

Preparation of nano-Ag/PAMPS composites. A certain amount of 2-acrylamido-2-methylpropane sulfonic acid (AMPS) (used as marketed) were dissolved in appropriate amount stilled water (used as marketed), then a certain amount of silver nitrate and potassium persulphate (KPS) were added respectively to the above solution. Subsequently, the mixture was stirred to be homogeneous, while $\mathrm{pH}$ of the solution was adjusted to 6.5 to 7.0 by $5 \mathrm{wt} \% \mathrm{NaOH}$ aqueous solution, so the mixing system was obtained. The microwave reactor (LWMC-201, Nanjing scientific research Co., Ltd., China; frequency, $2450 \mathrm{MHz}$; output power, $650 \mathrm{~W}$, tunable) was on while output of the microwave was adjusted to work efficiently. The reacting system became dusk after the microwave radiation for several minutes. The reaction was terminated when the polymerization of AMPS and reduction of the silver ion was finished. The reaction system was extracted and washed respectively by methanol and dimethyl formamide (DMF), and the brown powder was obtained. Finally, the products were subjected to vacuum drying at $100^{\circ} \mathrm{C}$ for hours for testing. The sample of pure AMPS polymer was also prepared by means of the similar polymerization approach in condition of adding no silver nitrate.

Characterization of nanosilver/PAMPS composites. The viscosity of PAMPS, which was prepared with different $\mathrm{pH}(\mathrm{pH}=1,4,7,10$ and 14, respectively) at reference condition, was measured with AR-G2 rotational rheometer (Thermal Analysis Co., USA) by way of the parallel plate with the shear rate of $0.125-125.0 \mathrm{sec}^{-1}$ in substep flow mode at $25^{\circ} \mathrm{C}$, the concentration of the sample was $0.366 \mathrm{~mol} / \mathrm{L}$. The UV spectrum of the samples was tested by UV-1600 dual-beam UV spectrophotometer (Beijing Rayleigh Instrument Co. Ltd., China). The morphology and size of nano-silver composites was observed by JEM-2010 High-Resolution Transmission Electron Microscopy (Japan Electronics Co., Ltd.). For Fourier transform infrared spectroscopies (FT-IR), the samples were pressed into pellet together with potassium bromide and were carried on a VECTOR-33 FT-IR 
spectrometer (Germany Bruker Co. Ltd.), with the wave number ranging from 500 to $4000 \mathrm{~cm}^{-1}$ at a resolution of $2 \mathrm{~cm}^{-1}$; X-ray powder diffraction (XRD) patterns of the nanocomposites were determined at a scanning rate of $0.03^{\circ} \mathrm{s}^{-1}$ in $2 \theta$ ranging from $10^{\circ}$ to $85^{\circ}$, using a X-ray diffractometer of XD-3 (Beijing General Instrument Co. Ltd.); X-ray photoelectron spectroscopy measurement were performed using a ESCALAB 250 spectrometer (USA Thermo Co. Ltd.); thermogravimetric analysis (TG) was conducted on a thermogravimetric analyzer (Thermal Analysis Co., USA) at a heating rate of $5^{\circ} \mathrm{C} / \mathrm{min}$ under nitrogen protection.

\section{Results and Discussion}

In order to research the optimal condition of pure AMPS polymerization, the viscosity of AMPS polymerizing system with different $\mathrm{pH}$ condition was conducted, based-on pertinence of viscosity of AMPS polymerizing system and AMPS polymerizing condition. The relation of viscosity of pure AMPS polymerizing system with $\mathrm{pH}$ was shown as Figure 1. The graph showed that the viscosity of the solution was increased with the increase of $\mathrm{pH}$ when $\mathrm{pH}$ was less than 7, subsequently, the viscosity of solution was decreased with $\mathrm{pH}$ of the polymerizing system, the viscosity of solution reaches to peak when the $\mathrm{pH}$ was about 7 , it suggested the neutral condition of polymerizing system was propitious to AMPS polymerizing, so the polymerizing condition was chosen as reaction of AMPS polymerizing accompanying with reducing of silver ion.

The infrared spectra of nanosilver/PAMPS composites were shown in Figure 2. It is given some characteristic peaks of the polymer, such as the $3437 \mathrm{~cm}^{-1}$ stretching vibration peak and the $1550 \mathrm{~cm}^{-1}$ bending vibration peak for $\mathrm{N}-\mathrm{H}$, the $2927 \mathrm{~cm}^{-1}, 1658 \mathrm{~cm}^{-1}, 1209 \mathrm{~cm}^{-1}, 1043 \mathrm{~cm}^{-1}$ and $629 \mathrm{~cm}^{-1}$ stretching vibration peak responding to $\mathrm{C}-\mathrm{H}, \mathrm{C}=\mathrm{O}$ group, $\mathrm{C}-\mathrm{N}$ group of sec-amide, $\mathrm{S}-\mathrm{O}$ and $\mathrm{C}-\mathrm{S}$ respectively. There is no stretching vibration peak for $\mathrm{C}=\mathrm{C}$. All those results showed that PAMPS as continuous phase of the nanocomposites was successfully prepared by microwave radiation.

To understand the shape of silver nanoparticles during the system reaction, UV-visible spectrometry of the nanocomposites during different reaction step were diagrammed in Figure 3. There is an absorption peak in 410 $\mathrm{nm}$ for nanosilver/PAMPS composites water-based system, which is analogous with that of Hiramatsu and Osterloh [19], who reported that the surface plasma resonance absorption peak of nearly spherical shape or spherical silver nanoparticles appeared in the vicinity of $425 \mathrm{~nm}$. The absorption peak of silver nanoparticles coated PAMPS polymer was not shifted with the microwave radiation time for composite reaction system, as shown in Figure 3, therefore, the morphology of silver nanoclusters was not affected by AMPS polymerization and microwave treatment. It hinted that silver nanoparticles were of nearly spherical shape or spherical structure in nanocomposites based on the variance of the width and strength of the absorption peak in the UV-visible spectra of nanocomposites, and it is identified too by TEM in following mentioned.

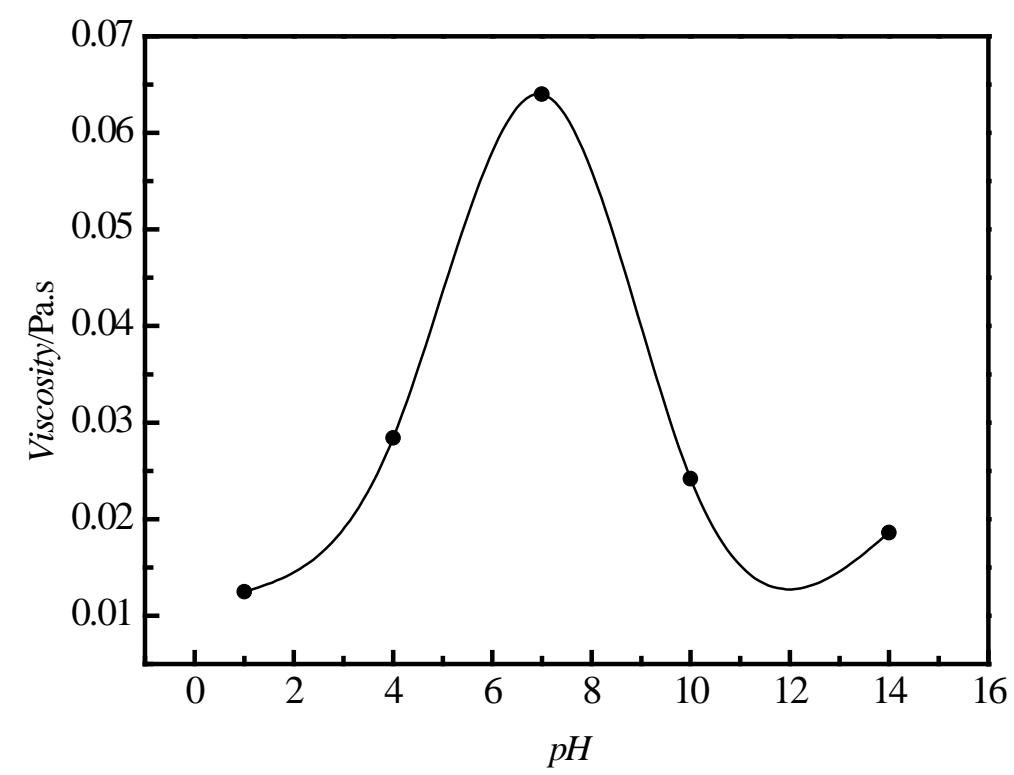

Figure 1. Relation of viscosity of PAMPS polymerizing system with $\mathrm{pH}$. 


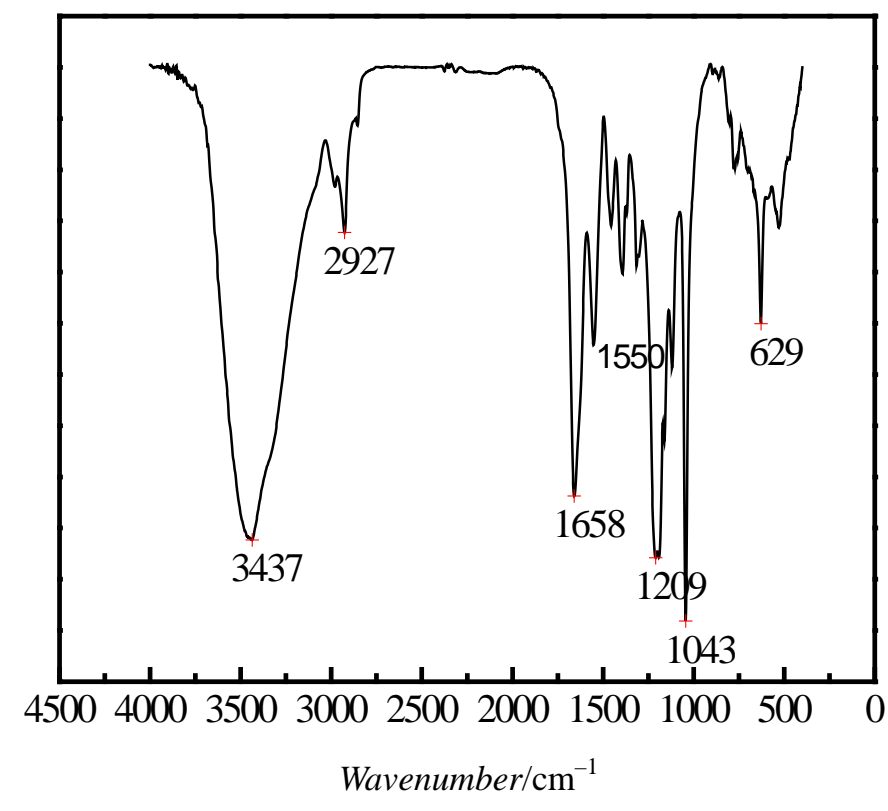

Figure 2. FTIR spectra of nano-Ag/PAMPS composite.

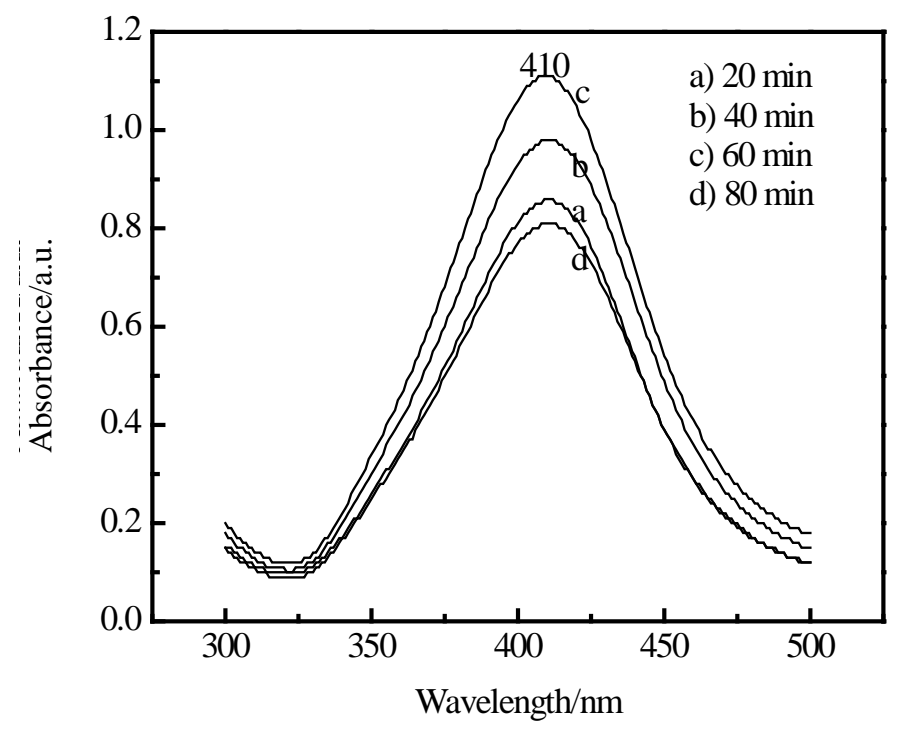

Figure 3. UV-Vis spectra of nano-Ag/PAMPS composite with different microwave time during reaction.

The XRD pattern of nano-Ag/PAMPS composites illuminated that there were four peaks appeared at $2 \theta=$ $37.99^{\circ}, 44.23^{\circ}, 64.59^{\circ}$ and $77.52^{\circ}$ respectively, which indicated that there were nano-silver crystal particulates within the nanocomposites. The crystal plane spacing (d) is $0.2367 \mathrm{~nm}, 0.2046 \mathrm{~nm}, 0.1442 \mathrm{~nm}$ and $0.1230 \mathrm{~nm}$ calculated by $2 d \sin \theta=\mathrm{n} \lambda$, corresponding to [111], [200], [220] and [311] crystal faces of silver, respectively, compared to PDFWIN\#87-0597. Therefore, the nanosilver particles possessed face-centered cubic structure within nanocomposites.

The silver nanoparticles within polymer composites were uniform in size with the range of $10-20 \mathrm{~nm}$, and were dispersed homogeneously in polymer matrix, shown as Figure 4. The silver nanoparticles were of nearly spherical shape or spherical structure, which is consistent with the results of UV-Vis identification above. The face-centered cubic polycrystalline structure was proved too by the electron diffraction analysis shown as right-up of Figure 4. According to the basic formula of electron diffraction $(\mathrm{d}=\mathrm{K} / \mathrm{R})$ and electron microscopy 


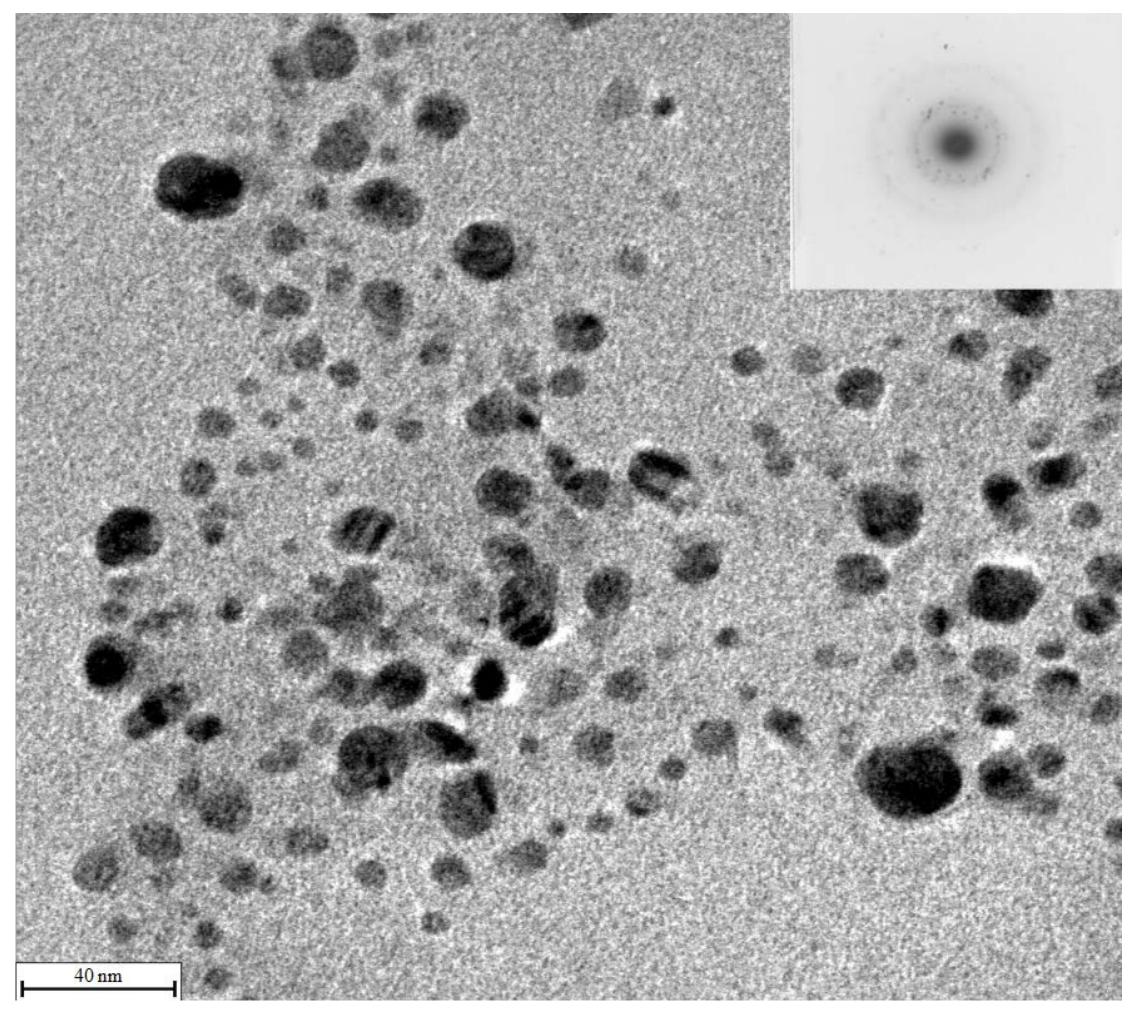

Figure 4. TEM photograph of nano-Ag/PAMPS composite.

constant $\mathrm{K}\left(2.008 \mathrm{~mm} \cdot \mathrm{nm}^{-1}\right)$, the corresponding crystal plane distances were calculated to be $0.237,0.205$, 0.145 and $0.123 \mathrm{~nm}$, which is consentaneous well with $d$ values of crystal plane distances of [111], [200], [220] and [311] (PDF card no. 87-0597).

To comprehend the interaction between nanosilver as dispersing phase and PAMPS polymer as continuous phase, the nano-Ag/PAMPS composites was examined by XPS, the survey spectrum of nanosilver/PAMPS is shown in Figure 5(a), it exhibited that the carbon, oxygen, nitrogen, sulphur, silver elements were found, which are consistent with the chemical components of the nanosilver/PAMPS composites.

In Figure 5(b), the energy spectrum peaks of $\mathrm{Ag}_{3 \mathrm{~d} 3 / 2}$ electrons positioned at $374.1 \mathrm{eV}$, which were increased up $1.1 \mathrm{eV}$ than the standard energy spectrum peaks of $\mathrm{Ag}_{3 \mathrm{~d} 3 / 2}$ electrons that positioned at $373.0 \mathrm{eV}$, that is, the energy spectrum peaks of $\mathrm{Ag}_{3 \mathrm{~d} 3 / 2}$ electrons within nanocomposites moved to the high binding energy, which indicated that the chemical environment of nanosilver clusters was changed already, it was possibly due to the coordination interaction between certain atoms of polymer and silver nanoparticles. The binding energy within the electronic shell was increased because of decreasing of valence electron density and the shielding effects of domestic electronic shell.

In the Figure 5(c) for $\mathrm{O}_{1 \mathrm{~s}}$ fitting spectra, the $\mathrm{O}_{1 \mathrm{~s}}$ binding energy $(531.4 \mathrm{eV}$ ) of $\mathrm{C}=\mathrm{O}$ was reduced $0.7 \mathrm{eV}$ than the standards $\mathrm{O}_{1 \mathrm{~s}}$ binding energy $(532.1 \mathrm{eV})$ of $\mathrm{C}=\mathrm{O}$, that is, the $\mathrm{O}_{1 \mathrm{~s}}$ binding energy moved to the lower region, which indicated that carbonyl oxygen atom accepting electronics cloud belonged to other atoms. In the Figure 5(d) for $N_{1 s}$ fitting spectra, the energy spectrum peaks of $N_{1 s}$ electrons positioned at $399.4 \mathrm{eV}$, which were lessened down $0.48 \mathrm{eV}$ than the standard energy spectrum peaks of $\mathrm{N}_{1 \mathrm{~s}}$ electrons that positioned at $399.88 \mathrm{eV}$, the $\mathrm{N}_{1 \mathrm{~s}}$ binding energy shift to lower region, which indicated that nitrogen atom accepted electronics cloud belonged to other atoms.

Based above discussing, it suggested that the electron density in the nitrogen atoms and carbonyl oxygen atoms in the PAMPS polymer was decreased, due to the transfer of electrons from silver particles that the binding energy was increased above mentioned, therefore the electron binding energy in the nitrogen atoms and oxygen atom shifts to a low binding energy level, as reported in the literature [20]. The specific interaction between nitrogen atoms and carbonyl oxygen atoms belonged to AMPS polymer and silver nanoparticles within the nanocomposites could be confirmed by the shift of the $\mathrm{N}_{1 \mathrm{~s}}$ and carbonyl $\mathrm{O}_{1 \mathrm{~s}}$ peaks to lower binding energy. 


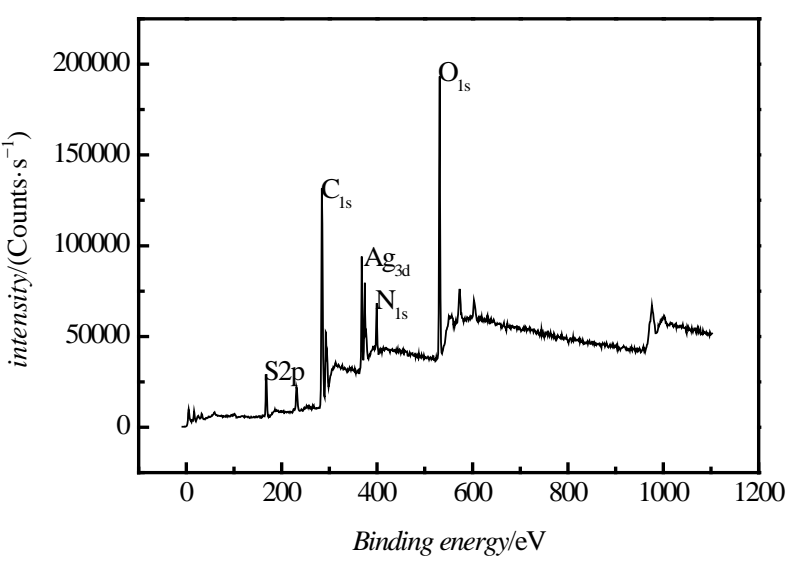

(a)

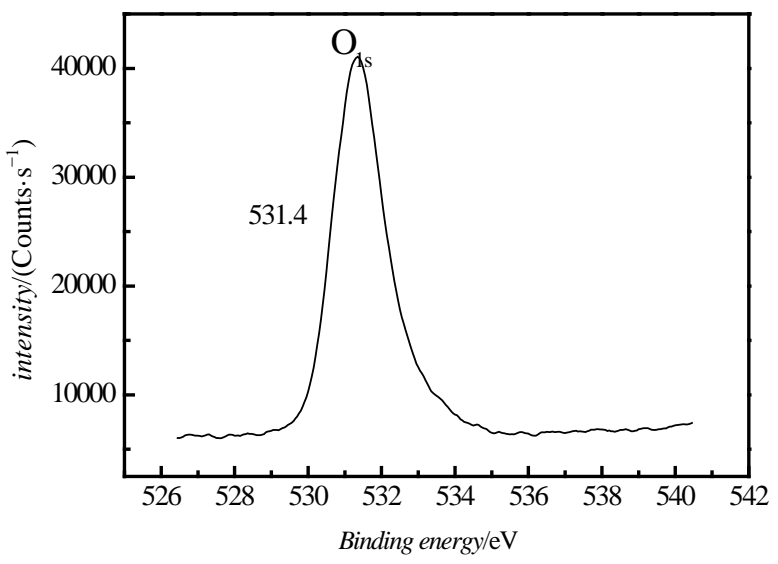

(c)

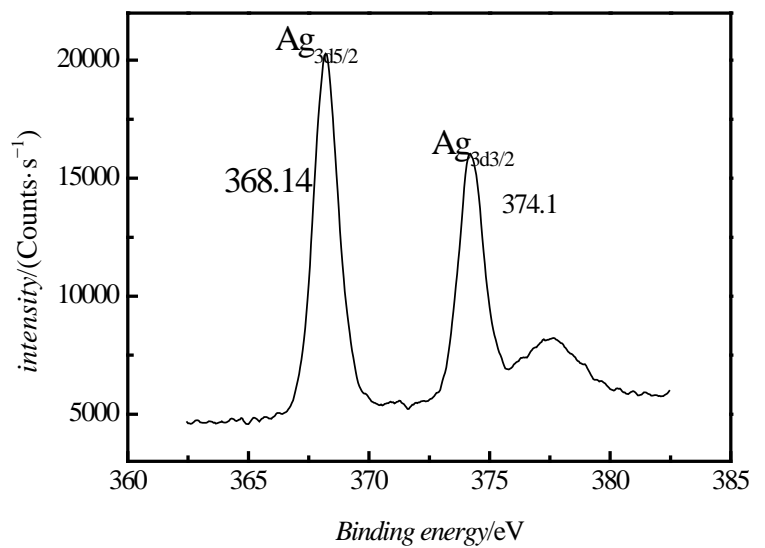

(b)

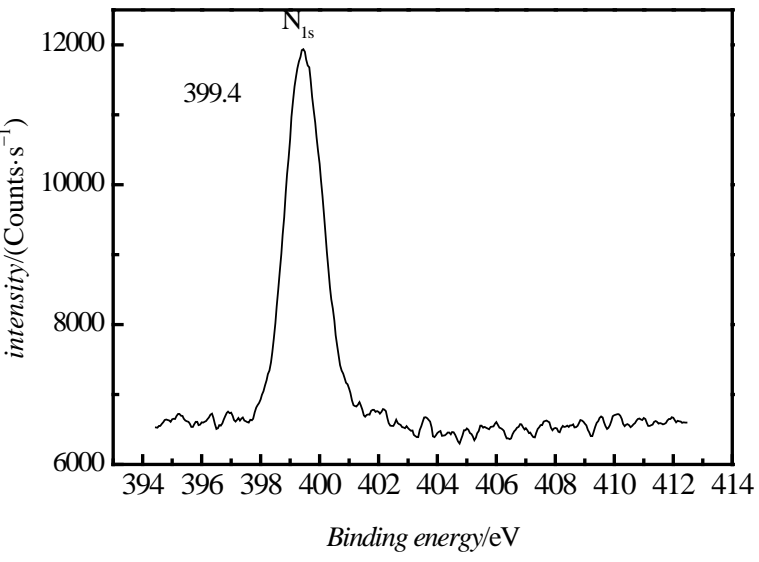

(d)

Figure 5. XPS spectra of nano-Ag/PAMPS composites.

To investigate the thermostability of the nanocoomposites, the thermogravimetric data of pure PAMPS and nanosilver/PAMPS composites was measured, shown as Figure 6. It interpreted that the decomposition temperature of PAMPS in the nanocomposites was $297^{\circ} \mathrm{C}$, which was lowered compared to $357^{\circ} \mathrm{C}$ of pure PAMPS, This is consistent with the result of Lee et al. [21], who showed the thermal stability of the polymer was reduced due to presence of silver particles in their nanocomposites. It was possibly that silver nanoparticles are homogeniously dispersed in polymer matrix to rapidly transmit heating effect and to promote decomposition of PAMPS polymer.

\section{Mechanism of Silver Ion Reduction with Nitrogen-Containing Compound by Microwave}

There is an interesting phenomenon that there was no changes in reaction system when sliver nitrate solution only without containing AMPS monomer by microwave, however, the color of reaction system was changed due to forming of silver nanoparticles in reaction system when adding PAMPS in reaction system after microwave radiation. It suggested that the silver ion was catalyzed to form silver nanopartilces by AMPS monomers. So the mechanism of silver nitrate reduced to nano-silver catalyzed with PAMPS by microwave was conjectured as following Figure 7.

The electron pair of nitrogen atom attached to sec-amine of AMPS molecular could coordinate with $\mathrm{Ag}^{+}$to form structure $\mathbf{1}$ (coordination ion). In high-frequency electric field of microwave, structure $\mathbf{1}$ was activated to come into being structure 2 (nitrogen radical cation) and silver atom when $\mathrm{Ag}^{+}$ion is reduced by attaining a electron of nitrogen atom of PAMPS, and PAMPS was transferred to form nitrogen radical cation. For nitrogen radical cation, it was very too unstable complex to comeback stable sec-amine structure, so the nitrogen atom 


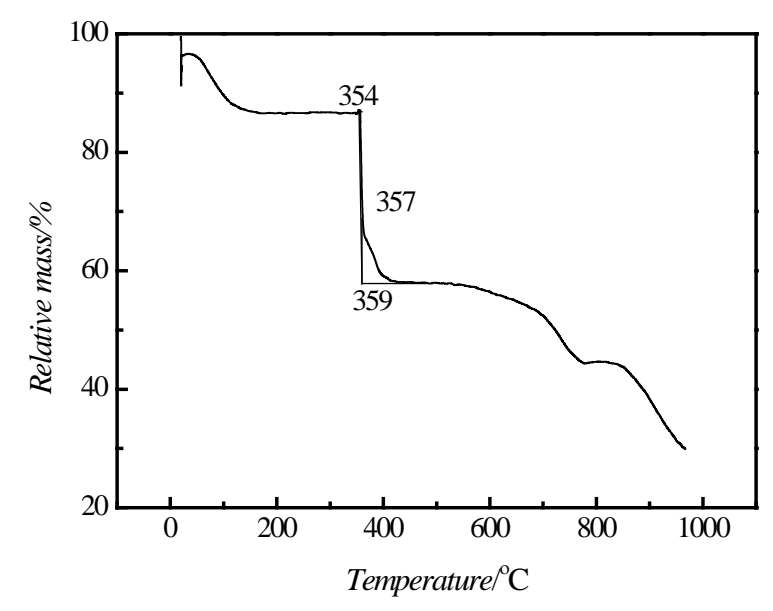

(a)

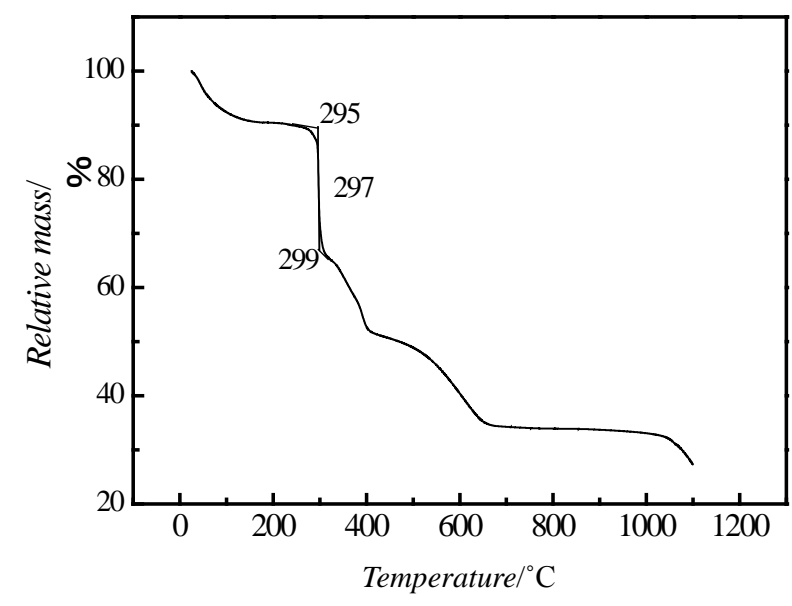

(b)

Figure 6. TG curve of (a) PAMPS and (b) nano-Ag/PAMPS composite.

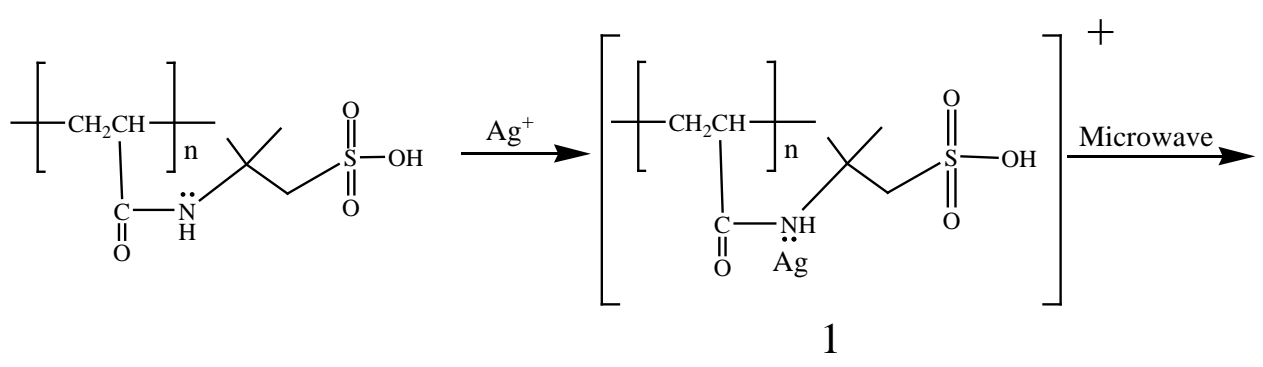<smiles>CC(C)(C)CC1C(=O)NC(C)(CS(=O)(=O)O)CC1(C)C(=O)O</smiles>

2

$$
+\mathrm{H}^{+}+\mathrm{OH} \cdot+\mathrm{Ag}(0)
$$

$\mathrm{n}$ Ag $(0) \longrightarrow(\mathrm{Ag}) \mathrm{n}$

$\mathrm{OH} \cdot+\mathrm{OH} \cdot \longrightarrow \mathrm{H}_{2} \mathrm{O}_{2}$

$2 \mathrm{H}_{2} \mathrm{O}_{2} \longrightarrow 2 \mathrm{H}_{2} \mathrm{O}+\mathrm{O}_{2}$

Figure 7. Diagram of silver nitrate reduced to nano-silver catalyzed with PAMPS by microwave.

attached to sec-amine of PAMPS played the role of intermediary for forming of silver nanoparticles. Also, nitrogen radical cation with low-energy could induce heavy water molecules $\left(\mathrm{D}_{2} \mathrm{O}\right)$ to be decomposed to deuterium radicals $(\mathrm{D} \cdot$ ) and deuterium hydroxyl radicals (.OD) in acetone heavy water solution, reported by Shi et al. [22]. So, here, nitrogen radical cation was produced once in reaction system and seized speedy a electron form water molecule and cause water molecular to be decomposed to hydrogen ion $\mathrm{H}^{+}$and hydroxyl radical $\cdot \mathrm{OH}$, meanwhile, nitrogen radical cation was come back sec-amine state in order to keep the structure stability 
of sec-amine. Based on this chemical reaction, forming of hydrogen ion resulted thereby in $\mathrm{pH}$ decreasing of reaction system, and it is consistent with the result of our experiment. In this way, with increasing of the chemical reaction, nanosilver particles were continuously generated with keeping the stable structure of PAMPS, in the end, nanosilver/PAMPS composite was formed.

\section{Conclusion}

The results showed that nanosilver/PAMPS composite was prepared in situ by microwave radiation. The UV-Vis spectrum revealed that the morphology of nanosilver particles was not affected by microwave treatment. TEM displayed that silver nanoparticles were homogeneously dispersed in polymer matrix and uniformed in size with the range of $10-20 \mathrm{~nm}$. XPS indicated that there was a chemical action between silver nanoparticles and nitrogen atoms and carbonyl oxygen atoms belonged to AMPS polymer within the nanocomposites. Thermoanalysis proved that the property of thermal stability of the PAMPS nanocomposites was decreasing with silver nanoparticles within composites. But also, the mechanism of silver ion which was reduced to nanosilver particles catalyzed by PAMPS under microwave was discussed.

\section{Acknowledgements}

This work was financially supported by the Natural Science Foundation of China (Grant No. 51173002).

\section{References}

[1] Chaudhary, V., Thakur, A.K. and Bhowmick, A.K. (2011) Improved Optical and Electrical Response in Metal-Polymer Nanocomposites for Photovoltaic Applications. Journal of Materials Science, 46, 6096-6105. http://dx.doi.org/10.1007/s10853-011-5573-x

[2] Gautama, A. and Ramb, S. (2010) Preparation and Thermomechanical Properties of Ag-PVA Nanocomposite Films. Materials Chemistry and Physics, 119, 266-271. http://dx.doi.org/10.1016/j.matchemphys.2009.08.050

[3] Vodnik, V.V., Bozanic, D.K., Dzunuzovic, E., Vukovic, J. and Nedeljkovic, J.M. (2010) Thermal and Optical Properties of Silver-Poly(methylmethacrylate) Nanocomposites Prepared by in-situ Radical Polymerization. European Polymer Journal, 46, 137-144. http://dx.doi.org/10.1016/j.eurpolymj.2009.10.022

[4] Dallas, P., Niarchos, D., Vrbanic, D., Boukos, N., Pejovnik, S., Trapalis, C. and Petridis, D. (2007) Interfacial Polymerization of Pyrrole and in situ Synthesis of Polypyrrole/Silver Nanocomposites. Polymer, 48, 2007-2013. http://dx.doi.org/10.1016/j.polymer.2007.01.058

[5] Yang, X.M., Li, L., Shang, S.M., Pan, G.L., Yu, X.H. and Yan, G.P. (2010) Facial Synthesis of Polypyrrole/Silver Nanocomposites at the Water Ionic Liquid Interface and Their Electrochemical Properties. Materials Letters, 64, 1918-1920. http://dx.doi.org/10.1016/j.matlet.2010.05.042

[6] Zapata, P.A., Tamayo, L., Páez, M., Cerda, E., Azócar, I. and Rabagliati, F.M. (2011) Nanocomposites Based on Polyethylene and Nanosilver Particles Produced by Metallocenic “in situ” Polymerization: Synthesis, Characterization, and Antimicrobial Behavior. European Polymer Journal, 47, 1541-1549. http://dx.doi.org/10.1016/j.eurpolymj.2011.05.008

[7] Zahoor, A., Qiu, T., Zhang, J.R. and Li, X.Y. (2009) Synthesis and Characterization of Ag@Polycarbazole Nanoparticles and Their Novel Optical Behavior. Journal of Materials Science, 44, 6054-6059. http://dx.doi.org/10.1007/s10853-009-3831-y

[8] Kamrupi, I.R., Phukonb, P., Konwerb, B.K. and Dolui, S.K. (2011) Synthesis of Silver-Polystyrene Nanocomposite Particles Using Water in Supercritical Carbon Dioxide Medium and Its Antimicrobial Activity. Journal of Supercritical Fluids, 55, 1089-1094. http://dx.doi.org/10.1016/j.supflu.2010.09.027

[9] Dallas, P., Sharma, V.K. and Zboril, R. (2011) Silver Polymeric Nanocomposites as Advanced Antimicrobial Agents: Classification, Synthetic Paths, Applications, and Perspectives. Advances in Colloid and Interface Science, 166, 119135.

[10] Cao, X.L., Cheng, C., Ma, Y.L. and Zhao, C.S. (2010) Preparation of Silver Nanoparticles with Antimicrobial Activities and the Researches of Their Biocompatibilities. Journal of Materials Science: Materials in Medicine, 21, 28612868. http://dx.doi.org/10.1007/s10856-010-4133-2

[11] Bang, J.H. and Suslick, K.S. (2010) Applications of Ultrasound to the Synthesis of Nanostructured Materials. Advanced Materials, 22, 1039-1059. http://dx.doi.org/10.1002/adma.200904093

[12] Spadaro, D., Barletta, E., Barrec, F., Curro, G. and Neri, F. (2010) Synthesis of PMA Stabilized Silver Nanoparticles by Chemical Reduction Process under a Two-Step UV Irradiation. Applied Surface, Science, 256, 3812-3816. 
http://dx.doi.org/10.1016/j.apsusc.2010.01.031

[13] Song, X.F., Lei, J., Li, Z.Y., Li, S.Y. and Wang, C. (2008) Synthesis of Polyacrylonitrile/Ag Core-Shell Nanowire by an Improved Electroless Plating Method. Materials Letters, 862, 2681-2684. http://dx.doi.org/10.1016/j.matlet.2008.01.014

[14] Anandhakumar, S. and Raichur, A.M. (2011) A Facile Route to Synthesize Silver Nanoparticles in Polyelectrolyte Capsules. Colloids and Surfaces B: Biointerfaces, 84, 379-383. http://dx.doi.org/10.1016/j.colsurfb.2011.01.029

[15] Li, S.-M., Jia, N., Ma, M.-G., Zhang, Z., Liu, Q.-H. and Sun, R.-C. (2011) Cellulose-Silver Nanocomposites: Microwave-Assisted Synthesis, Characterization, Their Thermal Stability, and Antimicrobial Property. Carbohydrate Polymers, 86, 441-447. http://dx.doi.org/10.1016/j.carbpol.2011.04.060

[16] Angshuman, P., Sunil, S. and Surekha, D. (2009) Microwave-Assisted Synthesis of Silver Nanoparticles Using Ethanol as a Reducing Agent. Materials Chemistry and Physics, 114, 530-532. http://dx.doi.org/10.1016/j.matchemphys.2008.11.056

[17] Yao, B.H., Xu, G.C., Zhang, H.Y. and Han, X. (2010) Synthesis of Nanosilver with Polyvinglpyrrolidone (PVP) by Microwave Method. Chinese Journal of Inorganic Chemistry, 26, 1629-1632.

[18] Xu, G.C., Shi, J.J., Li, D.J. and Xing, H.L. (2009) On Interaction between Nano-Ag and P(AMPS-co-MMA) Copolymer Synthesized by Ultrasonic. Journal of Polymer Research, 16, 295-299. http://dx.doi.org/10.1007/s10965-008-9229-8

[19] Hiramatsu, H. and Osterloh, F.E. (2004) A Simple Large-Scale Synthesis of Nearly Monodisperse Gold and Silver Nanoparticles with Adjustable Sizes and with Exchangeable Surfactants. Chemistry of Materials, 16, 2509-2511. http://dx.doi.org/10.1021/cm049532v

[20] Kim, D.H. and Jo, W.H. (2000) Studies on Polymer-Metal Interfaces. 2. Competitive Adsorption between Oxygen- and Nitrogen-Containing Functionality in Model Copolymers onto Metal Surfaces. Macromolecules, 33, 3050-3058. http://dx.doi.org/10.1021/ma982003q

[21] Lee, K.H., Rah, S.C. and Kim, S.G. (2008) Formation of Monodisperse Silver Nanoparticles in Poly(vinylpyrrollidone) Matrix Using Spray Pyrolysis. Journal of Sol-Gel Science and Technology, 45, 187-193. http://dx.doi.org/10.1007/s10971-007-1675-y

[22] Shi, H.B., Shao, C.L. and Yu, Z.L. (2001) Mechanism of the Reaction between Low Energy $\mathrm{N}^{+}$and $\mathrm{CH}_{3} \mathrm{COCH}_{3}$ with $\mathrm{D}_{2} \mathrm{O}$ as Solvent. Acta Physico-Chimica Sinica, 17, 986-990. 